\title{
Multiple myeloma masquerading as abducens palsy: A case report.
}

\author{
Gayle E LePosa ${ }^{1}$, M Adelita Vizcaino², Praveena Gupta ${ }^{3}$ \\ ${ }^{1}$ Wilmer Eye Institute, Johns Hopkins University School of Medicine, Baltimore, Maryland, United States \\ ${ }^{2}$ Department of Pathology, Johns Hopkins University School of Medicine, Baltimore, Maryland, United States \\ ${ }^{3}$ Department of Ophthalmology, University of Texas Medical Branch, Galveston, Texas, United States
}

\begin{abstract}
Purpose: To report a case of abducens palsy as the presenting symptom of multiple myeloma Case report: A 68-year-old woman with a history of systemic hypertension presented complaining of constant horizontal diplopia in left gaze for six weeks. Her entering acuities were 20/25 in the right eye and 20/20 in the left eye. Extraocular muscle testing revealed left sixth nerve palsy without any misalignment in primary gaze. The remainder of her ophthalmic examination, including other neurological testing such as cranial nerve testing, pupils, and confrontation fields, was unremarkable. MRI of the brain and orbits indicated numerous osseous lesions throughout the skull with a high-risk lesion invading the left cavernous sinus and encasing both the left internal carotid artery and the left optic nerve. After receiving these results, she was referred urgently for further imaging, which revealed additional lesions throughout her axial and appendicular skeleton, including other high risk lesions in the thoracic spine and the pelvis. Further workup confirmed the diagnosis of multiple myeloma.

Conclusions: Ophthalmic findings related to multiple myeloma are generally rare and tend to occur late in the course of known disease. In this case, ipsilateral isolated sixth nerve palsy was the presenting sign of multiple myeloma in a patient. Although neurological etiology is not common with sixth nerve palsies, neurological workup including imaging should be considered routinely in patients who present with isolated sixth nerve palsy, particularly when vasculopathic risk factors are not suggested by patient history.
\end{abstract}

Keywords: Multiple myeloma, Lisinopril, Gaze, Oculomotor palsies, Lesion biopsy.

Accepted on November 23, 2017

\section{Introduction}

Abducens nerve involvement is the most common neuroophthalmic complication seen in multiple myeloma, however it has been described as the presenting symptom that lead to the eventual diagnosis of multiple myeloma only a handful of times[1-7]. Sixth nerve palsy is more frequently seen in conjunction with other neurological findings on examination (such as other oculomotor palsies) or after treatment has been initiated [4, 8-14] We describe a patient who presented with an abducens nerve palsy in the setting of a vasculopathic risk factor who was later diagnosed with advanced multiple myeloma.

\section{Case Report}

A 68 year old African American female presented to our clinic complaining of constant horizontal diplopia in left gaze for the past six weeks. She denied any pain or discomfort with the double vision. Her medical history was remarkable for hypertension, which was treated with lisinopril. Ophthalmic examination revealed entering acuities of $20 / 25$ in the right eye and 20/20 in the left eye. Pupils were equal and reactive to light without afferent pupillary defect. Confrontation fields were normal. Extraocular muscle movements revealed a left abduction deficit without misalignment in primary gaze. The patient endorsed horizontal diplopia only in left lateral gaze, and denied any diplopia in primary gaze. She reported symmetric sensation bilaterally along the fifth cranial nerve V1, V2, and V3 distributions. The seventh cranial nerve was intact with symmetric smile and the eighth cranial nerve was intact to finger rub bilaterally. The palpebral fissures were symmetric at $11 \mathrm{~mm}$ bilaterally. Slit lamp examination was remarkable for symmetric nuclear sclerosis bilaterally but otherwise unremarkable. Dilated fundus examination was unremarkable in both eyes. Differential diagnoses included giant cell arteritis, myasthenia gravis, Lyme disease, cancer, thyroid disease, small aneurysm, and ischemic disease. Upon further questioning, she denied any recent weight loss, scalp tenderness, jaw claudication, and had good temporal artery pulses bilaterally, which was less suspicious for giant cell arteritis. She also denied any weakness of her extremities, hoarse voice, or difficulty swallowing, which was less suspicious for myasthenia gravis. She was also asked if she had any recent tick bites or if she spent a great deal of time outdoors, especially in heavily wooded areas. She denied both, which lowered our suspicion for Lyme disease. Magnetic resonance imaging (MRI) of the brain and orbits with and without contrast was ordered and the patient was instructed to return within six weeks or sooner with any change in her symptoms. 
Citation: G E LePosa, M A Vizcaino, P Gupta. Multiple myeloma masquerading as abducens palsy: A case report. J Clin Ophthalmol. 2017;1(1):23-26.

Six weeks later, the patient completed the MRI imaging and returned to our clinic for follow up evaluation the same day. The imaging results indicated numerous osseous lesions in the calvarium, skull base, and maxillofacial bones highly suspicious for metastatic disease, notably with a large lesion in the clivus with invasion of the left cavernous sinus, encasing both the left internal carotid artery 360 degrees and the left retro-orbital optic nerve, and approaching the left orbital apex without evidence of intraorbital extension (Figure 1). A discrete mass was also identified within the left orbital roof, causing buckling of the superior rectus (Figure 2). After reviewing the results, the interpreting radiologist was contacted promptly to further discuss the patient. Given the extensive findings seen on the MRI of the brain and orbits, the radiologist recommended immediate referral to the emergency department for stabilization of the neck and further imaging, given the high potential for lesions with the cervical spine which could be at risk for pathological fracture. The patient was admitted and underwent additional imaging, which indicated numerous lesions throughout her axial and appendicular skeletons. Lesions at high risk for fracture were found at T5, causing spinal cord compression, and at the left femoral neck and right humerus. Serum protein electrophoresis was normal however serum free light chain analysis revealed overproduction of lambda light chains relative to kappa light chain synthesis, which is commonly seen in patients with multiple myeloma or amyloidosis. Histologic sections of the femoral biopsy showed diffuse infiltration by numerous plasma cells characterized by eccentric nuclei with heterochromatin in a characteristic "cartwheel" arrangement and basophilic cytoplasm with a perinuclear pale zone containing an extensive Golgi complex. The neoplastic cells showed kappa light chain restriction by immunohistochemistry, with no lambda light chain expression (Figure 3 ).

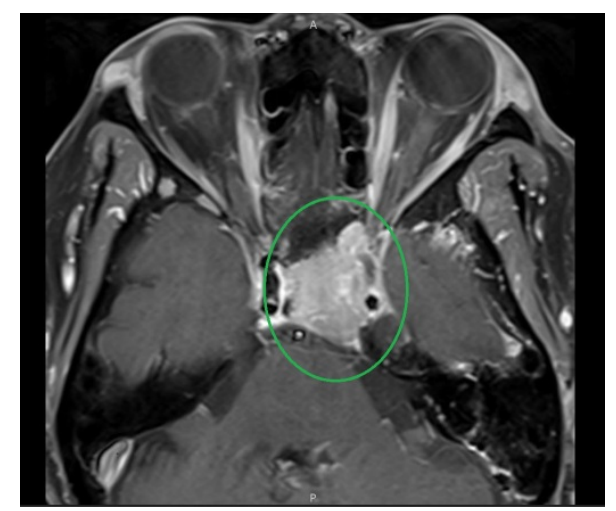

Figure 1. A T1-weighted axial view at the level of the cavernous sinus demonstrating a metastatic lesion (encircled) within the left cavernous sinus extending into the left orbital apex.

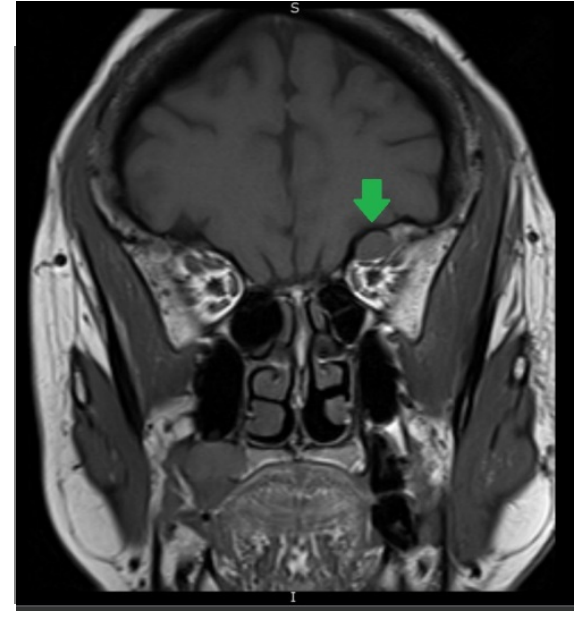

Figure 2. A T1-weighted coronal view of the orbits demonstrating a metastatic lesion (arrow) invading through the left orbital roof and impinging on the left superior rectus.

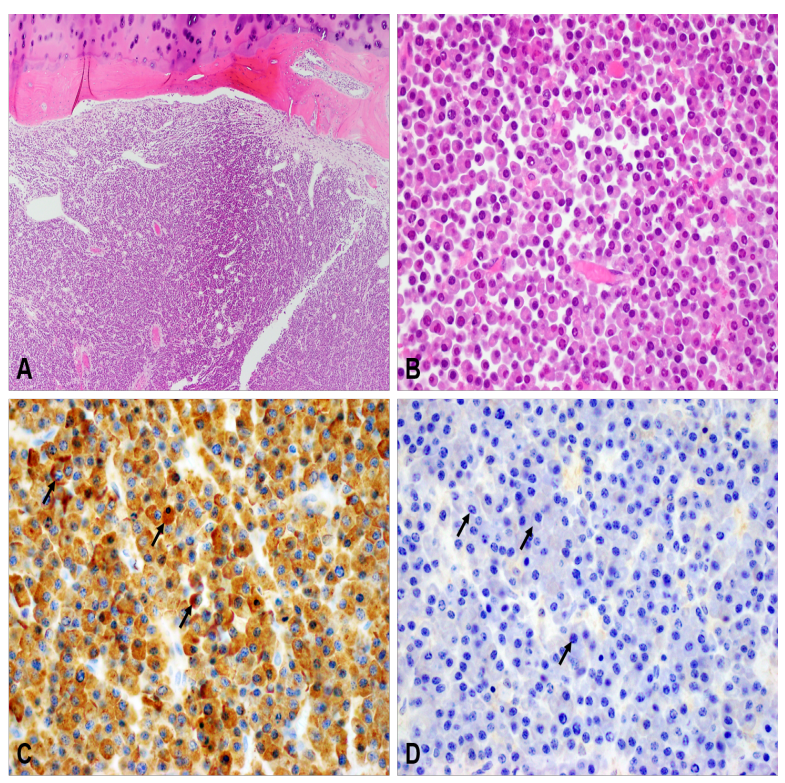

Figure 3. $A, B$. The femoral tissue is replaced by a diffuse, monomorphic population of neoplastic plasma cells. $C$. Immunohistochemical stains demonstrate strong and diffuse expression for kappa light chain (arrows). D. In contrast, lambda light chain immunoreactivity is negative (arrows).

\section{Discussion}

Our patient presented complaining of new onset diplopia only in left gaze, and her ophthalmic exam was unremarkable for other neurological findings. After MRI, lab workup, and lesion biopsy, she was formally diagnosed with multiple myeloma. During her visit with ophthalmology, she denied fatigue and lumbar pain, which is atypical of patients who diagnosed with multiple myeloma. Other similar case reports of patients presenting with a sixth nerve palsy that subsequently led to the diagnosis of multiple myeloma differ from our patient in that she did not present with other concurrent visual symptoms, such as transient blurring of vision [15], headache or occipital pain [16]. Unfortunately, although our patient has been scheduled for follow up in our ophthalmology clinic numerous times, she has failed to return for further evaluation. She is 
currently being followed by oncology, where she has received treatment with a proteasome inhibitor, an additional immunomodulatory medication, and a steroid.

Multiple myeloma is characterized as a plasma cell dyscrasia that arises when the bone marrow produces abnormally high levels of plasma cells. It is typically seen in patients between the ages of 40 and 70 with the median age at the time of diagnosis being 69 years. It has a slightly higher predilection for males versus females, and the frequency of multiple myeloma is 2-3 times higher in African Americans than any other ethnic group [17]. Presenting symptoms are usually nonspecific and vague, including fatigue, weight loss, and bone pain with movement. Lab testing in patients with multiple myeloma frequently demonstrates Bence Jones proteinuria, renal insufficiency, normocytic normochromic anemia, and hypercalcemia [16]. Ophthalmic manifestations of multiple myeloma are uncommon and irregular, and can affect all ocular structures. Crystalline and copper deposits may be seen in the cornea, and band keratopathy may occur secondary to hypercalcemia. In the posterior pole, retinal hemorrhages, nerve fiber layer infarcts, and papilledema may be present. Microcysts of the uveal tract and orbital tumors have also been documented. These orbital tumors often involve the orbital roof and frontal bones causing proptosis, and even infiltration of the extraocular muscles in once case. Cranial nerve palsies may also be present. Neuro-ophthalmic manifestations may result from hematologic effects, compression, or direct infiltration of the cranial nerves or visual pathway. Multiple cranial nerve palsies are more likely to occur than an isolated palsy, believed to be secondary to compression from basal skull lesions [1-3].

When facing a suspected isolated sixth nerve palsy, it is important to consider differential diagnoses that may mimic its symptoms. These include thyroid-related ophthalmopathy, myasthenia gravis, orbital inflammatory pseudotumor, Duane's retraction syndrome type 1 , Mobius syndrome, orbital fracture with medial rectus entrapment, and spasm of the near reflex [15].

Management of multiple myeloma aims to curtail the proliferation of plasma cells, as there is no cure. Considerations for treatment include patient age, presence of other comorbidities, and the presence of end-organ disease. Patients without end-organ damage are diagnosed with smoldering multiple myeloma and do not require treatment [18] Immunomodulatory drugs, anti-neoplastic agents and proteasome inhibitors, sometimes alongside dexamethasone, have become the first line of treatment for patients. Autologous stem cell transplantation can prolong survival significantly in patients with multiple myeloma but can only be utilized in a certain subset of patients: those up to 65-70 years of age, those without heart, lung, kidney, or liver dysfunction, and those without other uncontrolled comorbidities (e.g., diabetes mellitus). Allogenic stem cell transplantation has been used for several decades however its usefulness is limited secondary to complications from graft-versus-host disease [19] Treatment of patients with relapsed or progressing disease can be challenging, especially as most patients eventually become drug resistant. Novel therapies for the treatment of relapsed multiple myeloma include a new proteasome inhibitor, a new immunomodulatory drug, monoclonal antibodies, signal transduction modulators, and histone deacetylase inhibitors [7]. Radiation is regularly used for the management of plasmacytomas. The secondary effects of multiple myeloma must also be treated, and includes the use of bisphosphonates (along with cholecalciferol and calcium) to reduce pathological vertebral fractures and bone pain [4]. Reduced kidney function is commonly seen in patients with multiple myeloma, and may occur under several different processes. High concentrations of free light chains can lead to direct damage of the kidney tubules. Kidney damage can also occur via plasma cell infiltration, hyperuricemia, hypercalcemia, hypovolemia, renal amyloidosis, and the use of nephrotoxic agents. Plasmapheresis and free light chain removal via hemodialysis should be considered in patients who are high risk for progressive kidney damage [20].

Although a sixth nerve palsy is often secondary to a nonthreatening etiology and can spontaneously resolve, they can also be associated with a neurological process which can benefit from early diagnosis and treatment. Imaging should be considered as part of any routine evaluation in a patient with a sixth nerve palsy, as our patient demonstrates that neurological processes may not always present with multiple neurological symptoms and early detection can be critical for these patients.

\section{Acknowledgment}

The authors have no financial disclosures to report.

\section{References}

1. Na JH, Park SH, Shin SY. Multiple myeloma manifesting as a fluctuating sixth nerve palsy. Korean J Ophthalmol 2009;23:232-3

2. Jiang $\mathrm{C}, \mathrm{Lin} \mathrm{Q}, \mathrm{Wu} \mathrm{X}$, et al. Sellar solitary plasmacytoma progressing to multiple myeloma: A case report and literature review. Medicine (Baltimore) 2014;93:e58.

3. Zahavi A, Manor RS, Lahav M, et al. Isolated sixth nerve palsy--presenting sign of multiple myeloma. Harefuah 2013;152:121.

4. Krawczyk J, Meenaghan T, Kelly M, et al. A case series of sixth nerve palsy associated with intracranial plasmacytoma. Haematologica 2009;94:623.

5. Hale AN, Wasik AJ. Isolated sixth cranial nerve palsy as a complication of multiple myeloma. Clin Refractive Optom 2006; 17:182-8

6. Feletti A, Tartaglia M, Campanini B, et al. Isolated oculomotor palsy as initial manifestation of multiple myeloma. J Fr Ophtalmol 1995;18:771-6.

7. Seki Y, Koga A, Fukai S, et al. Etiological study on 6 cases of abducens paralysis. Folia Ophthalmol Jpn 1977;28:18-22.

8. Thiruvengadam SS, Prayson RA. Multiple myeloma presenting with unilateral abducens and trigeminal nerve palsies. J Clin Neurosci 2016;26:143-4. 
Citation: G E LePosa, M A Vizcaino, P Gupta. Multiple myeloma masquerading as abducens palsy: A case report. J Clin Ophthalmol. $2017 ; 1(1): 23-26$.

9. Cetin G, Cem Ar M, Cerit A, et al. A rare entity in multiple myeloma: Six nerve paralysis. Indian J Hematol Blood Transfus. Indian $\mathrm{J}$ Hematol Blood Transfus 2014;30:70-2.

10. Grisold A, Weber C, Gisslinger $\mathrm{H}$, et al. MRI negative meningeal myelomatosis with bilateral abducens nerve palsy and response to intrathecal chemotherapy. Eur J Neurol. 2014;21:273.

11. Chen K, Hsu SPC, Chen M, et al. Multiple myeloma with cavernous sinus involvement. Kaohsiung $\mathrm{J}$ Med Sci 2013;29:517-8.

12. Kashyap R, Kumar R, Kumar S. Cranial nerve palsy in multiple myeloma and solitary plasmacytoma. Asia-Pac J Clin Oncol 2010;6:251-5.

13. Neki NS, Sharma RK, Sharma N, et al. Multiple myeloma presenting as proptosis and sixth nerve palsy. J Assoc Physicians India 2001;49:1116-7.

14. Kanoh T, Okuda T, Hayashi M, et al. Multiple myeloma presenting as parasellar syndrome and cranial nerve palsies. Rinsho Ketsueki 1996;37:260-4.

15. Chakrabarti S, Pan K, Panchadhyayee S, et al. Multiple myeloma presenting as isolated sixth cranial nerve palsy. Int J Res Med Sci 2014;2:738-40.

16. Movsas TZ, Balcer LJ, Eggenberger ER, et al. Sixth nerve palsy as a presenting sign of intracranial plasmacytoma and multiple myeloma. J Neuroophthalmol. 2000;20:242-5.

17. Azarmina M, Azarmina H. The six syndromes of the sixth cranial nerve. J Ophthalmic Vis Res 2013;8:160-71.

18. Rollig C, Knop S, Bornhauser M. Multiple myeloma. Lancet 2015;385:2197-208.

19. El Amm J, Tabbara IA. Emerging therapies in multiple myeloma. Am J Clin Oncol 2015;38:315-21.

20. Grzasko N, Morawska M, Hus M. Optimizing the treatment of patients with multiple myeloma and renal impairment. Clin Lymphoma Myeloma Leuk 2015;15:187-98.

\section{*Correspondence to}

Gayle E. LePosa, OD, FAAO

Wilmer Eye Institute

Johns Hopkins University School of Medicine

Maryland, United States

Tel: 410-955-5650

E-mail: glowe2@jhmi.edu 